\title{
Characteristics of Hepatitis B and D Co-infection: A Descriptive Study
}

\author{
Mahbobeh Hajiabdolbaghi ${ }^{1}$, Zahra Abdiliae ${ }^{2}$, Jaleh Bayani ${ }^{3}$, Mehdi Qaempanah ${ }^{4}$ and Fereshteh \\ Ghiasvand ${ }^{5,{ }^{*}}$ \\ ${ }^{1}$ Department of Infectious Diseases, Iranian Research Center for HIV/AIDS, Iranian Institute for Reduction of High Risk Behaviors, Tehran University of Medical Sciences, \\ Tehran, Iran \\ ${ }^{2}$ Department of Infectious Disease, Tehran University of Medical Sciences, Tehran, Iran \\ ${ }^{3}$ Hepatitis Clinic, Tehran University of Medical Sciences, Tehran, Iran \\ ${ }^{4}$ School of Medicine, Tehran University of Medical Sciences, Tehran, Iran \\ ${ }^{5}$ Department of Infectious Diseases, Liver Transplantation Research Center, Imam Khomeini Hospital Complex, Tehran University of Medical Sciences, Tehran, Iran \\ "Corresponding author: Department of Infectious Diseases, Liver Transplantation Research Center, Imam Khomeini Hospital Complex, Tehran University of Medical Sciences, \\ Tehran, Iran. Tel: +98-9121828679, Fax: +98-2166581598, Email: ghiasvandfereshte@gmail.com
}

Received 2020 February 08; Revised 2020 November 16; Accepted 2021 January 19.

\begin{abstract}
About $5 \%$ of cases with chronic hepatitis B virus (HBV) are co-infected with hepatitis D virus (HDV), and this co-infection possesses a high risk for hepatocellular carcinoma (HCC) and cirrhosis. We aimed to evaluate the epidemiological and histopathological characteristics and response to treatment in patients with HBV and HDV co-infection in a hepatitis clinic in Tehran, Iran. In this study, 80 patients were enrolled and evaluated for age, sex, degree of liver fibrosis, virologic status, and response rate. The incidence of coinfection of hepatitis B and D was 4.9\% (80/1,631 HBV-infected cases) in our clinic. Thirty-seven (46.25\%) patients had liver cirrhosis, and four (5\%) patients had HCC. Besides, HDV Ribonucleic Acid (RNA) polymerase chain reaction (PCR) was positive for 31 (38.75\%) patients. Among 30 patients, 14 (46.6\%) completed interferon therapy (for equal to or more than 48 weeks) and had a response with undetectable HDV RNA PCR after treatment. According to our results, for cirrhotic patients in HBV/HDV co-infection that was about $46 \%$, it seems that the early detection of HDV antibody (Ab) plays a significant role in the prevention of progressive liver failure, and treatment with interferon can help these patients by improving the prognosis.
\end{abstract}

Keywords: Hepatitis B Virus, HBV, Hepatitis Delta Virus, HDV, Cirrhosis

\section{Background}

Hepatitis D virus (HDV) is a defective, single-stranded ribonucleic acid (RNA) virus that depends on the supply of hepatitis B surface antigen (HBsAg) for its assembly and transmission. The HDV superinfection with chronic hepatitis $\mathrm{B}$ can lead to different clinical manifestations in patients (1). About $10.58 \%$ of chronic hepatitis B virus (HBV) patients are co-infected with $\operatorname{HDV}(2,3)$, meaning that around 62 - 72 million people are HDV carriers worldwide (4). However, in different geographical regions, the prevalence of HDV infection varies significantly (5).

In HBV and HDV co-infection, the role of HDV infection is still unknown (6), and prognostic factors for HDV infection are uncertain too (7). Chronic HBV and HDV coinfection imposes a greater risk of developing cirrhosis and hepatocellular carcinoma (HCC), compared to HBV infection alone (8-10). The rate of hepatitis B in Iran is estimated at $2.14 \%$ (2); however, co-infection with hepatitis B and $\mathrm{D}$ is different.

\section{Objectives}

We aimed to evaluate the epidemiological and histopathological characteristics and response to treatment in patients with HBV and HDV concurrent infection in a hepatitis clinic in Tehran.

\section{Methods}

\subsection{Participants}

In this retrospective observational cross-sectional study, patients with hepatitis B and D co-infection were referred from 2004 to 2018 to the hepatitis clinic of Imam Khomeini Hospital, Tehran, Iran. The inclusion criteria were the confirmation of chronic hepatitis $\mathrm{B}$ infection based on positive HBsAg for more than six months and hepatitis D simultaneously with the serologic method (total IgM and IgG antibodies). The exclusion criteria were infection with human immunodeficiency virus (HIV), presence of alcoholic, autoimmune, and drug-induced hepatitis, lack of serological evidence to determine the 
hepatitis B infection, and loss of data to make the analysis possible.

\subsection{Data Gathering and Treatment}

The gathered data included demographic and laboratory data, as well as the degree of liver fibrosis, virologic status, and response rate. Demographic characteristics including age, gender, year of birth, date of diagnosis of hepatitis B infection, date of treatment onset, and history of hepatitis D infection were registered. Laboratory data were collected based on evidence of liver cirrhosis. The degree of liver fibrosis was confirmed by pathologic findings in a liver biopsy defined by the METAVIR score or Fibroscan. The virologic status was determined using the molecular method of polymerase chain reaction (PCR) with the COBAS TaqMan method (Roche) whose lower limit of detection for COBAS TaqMan was 6 IU/mL. Interferon- $\alpha$ (PEG INF$\alpha 180$ microgram once a week injection) was prescribed for all patients with positive HDV viral load or undetectable HDV and HBV with significant fibrosis. Finally, response to treatment within 48 weeks of the initiation of treatment based on the reduction of hepatitis D virus loading was evaluated by using the PCR molecular method at the end of treatment. Nonresponding patients were defined by a reduction in the HDV PCR viral load by less than one logarithm.

\subsection{Ethical Considerations}

Following the Declaration of Helsinki, written informed consent was obtained from all participants before the initiation of the study. The study protocol was approved by the institutional review board of Tehran University of Medical Sciences.

\subsection{Measurements and Statistical Analysis}

The analysis was performed using SPSS software (IBM SPSS Statistics for Windows, version 25.0. Armonk, NY: IBM Corp). Quantitative variables were reported by mean and standard deviation (SD) and qualitative variables were reported using frequency and percentage.

Because of the normal distribution of data, an independent t-test was used to assess the means differences. The chi-square and Fisher exact tests were used to assess the statistical relationships between categorical variables. The level of significance was set at a P-value $<0.05$ for all analyses.

\section{Results and Discussion}

Among 1,631 HBV-infected patients, 80 patients were included as hepatitis B and D co-infection. The incidence of hepatitis B and D co-infection was 4.90\% (80/1,631 HBV infection cases). The mean age was $45.69 \pm 15.10$ years (range: 17 - 78). The numbers of males and females were $52(65 \%)$ and $28(35 \%)$, respectively. In terms of nationality, 66 (82.50\%) were from Iran, 13 (16.25\%) from Afghanistan, and one (1.25\%) from Azerbaijan. Demographic and laboratory characteristics of $\mathrm{HBV} / \mathrm{HDV}$ co-infected patients are presented in Table 1. Liver cirrhosis was found in 37 (46.25\%) patients.

Table 1. Demographic and Laboratory Characteristics of HBV/HDV Co-infected Patients $^{\mathrm{a}}$

\begin{tabular}{|c|c|}
\hline & Values \\
\hline Age, $y$ & $45.5 \pm 14$ \\
\hline Alanine transaminase, units/L & $72.3 \pm 63.7$ \\
\hline Aspartate transaminase, units/L & $69.2 \pm 56$ \\
\hline International normalized ratio & $1.2 \pm 0.25$ \\
\hline Creatinine, $\mathbf{m g} / \mathrm{dL}$ & $0.94 \pm 0.25$ \\
\hline Bilirubin, total, mg/dL & $1.33 \pm 0.99$ \\
\hline Bilirubin, conjugated, mg/dL & $0.50 \pm 0.55$ \\
\hline White blood cell count, $\times 10^{3} / \mathrm{mm}^{3}$ & $6.03 \pm 5.03$ \\
\hline Hemoglobin, $\mathrm{g} / \mathrm{dL}$ & $13.9 \pm 1.7$ \\
\hline Platelet, $\mathbf{1 0}^{3} / \mathrm{L}$ & $148.2 \pm 78.4$ \\
\hline Positive hepatitis B e-antigen (HBe Ag positive) & $7(8.75)$ \\
\hline $\begin{array}{l}\text { Positive hepatitis B virus (HBV) polymerase chain } \\
\text { reaction (PCR) }\end{array}$ & $44(55)$ \\
\hline $\begin{array}{l}\text { Positive hepatitis delta virus (HDV) polymerase chain } \\
\text { reaction (PCR) }\end{array}$ & $25(23.18)$ \\
\hline
\end{tabular}

The mean Fibroscan score in patients with HBV and HDV co-infection was 18.25 kilopascal (KP) (range: 6 - 62). Liver biopsy was performed for $15(18.75 \%)$ patients, and the results were as follows: Stage $\leq 2$ (no fibrosis) in six (40\%) patients, stage 3 in four (26.66\%) patients, and stage $\geq 4$ in five (33.33\%) patients. Hepatocellular carcinoma (HCC) was evidenced in four (5\%) patients. Positive hepatitis B eantigen (HBeAg) was found in seven (8.75\%) patients.

In terms of HBV DNA viral load, 44 (55\%) patients were positive. The HBV DNA of 25 (23.18\%) patients was $\leq 2000$ IU/mL; 11 (25\%) patients had 2000 - $20000 \mathrm{IU} / \mathrm{mL}$, and eight (18.18\%) patients had a viral load above 20,000 IU/ML. The HDV RNA PCR was positive for 31 (38.75\%) patients.

Thirty $(37.50 \%)$ patients were treated with interferon, among whom, in eight (26.66\%) patients, treatment was switched to oral tenofovir. Also, 29 patients received only oral agents for the treatment of chronic hepatitis $\mathrm{B}$. Besides, $14(14 / 30,46.66 \%)$ patients completed interferon therapy (for at least 48 weeks) and had a response with undetectable HDV RNA PCR after treatment, and none of them 
were cirrhotic. Moreover, 16 (16/30, 53.33\%) patients had the disruption of interferon after 3 - 6 months because of following reasons. Five $(5 / 30,16.66 \%)$ patients had no response to treatment. Four $(4 / 30,13.33 \%)$ patients experienced the complications of interferon (neutropenia, a rise in liver enzymes, myalgia, decompensated cirrhosis). Seven patients $(7 / 30,23.33 \%)$ did not come to the clinic to continue treatment.

Seven (8.75\%) patients had co-infection with hepatitis C virus (HCV); two of them received special treatment, and five had undetectable HCV PCR. Four patients had a cirrhotic liver. There was no significant correlation between hepatic cirrhosis and HBV DNA viral load (P-value 0.429). However, there was a significant correlation between liver cirrhosis and HDV RNA viral load (P-value: 0.009).

There are few studies on HBV and HDV clinical manifestations, treatment, associated factors, lab data, prognosis, and factors effective in the disease process. Miao et al. (11) conducted a meta-analysis with a random-effects model to evaluate the global prevalence, disease progression, and clinical outcomes of hepatitis delta virus infection. They reported a global prevalence of HDV infection to be $0.80 \%$ in the general population and $13.02 \%$ in the carriers of hepatitis B virus surface antigen (HBsAg). Also, this study reported a high prevalence of HDV infection among HBV-HCV co-infected people (11). In the current study, we reported the incidence of co-infection with hepatitis B and D to be $4.9 \%$ (80/1,631 HBV-infected cases) in our clinic, which seems to be less than the rate in this meta-analysis. Seven of our patients had HCV and HBV co-infection. The rate of HDV co-infection is variable in different settings. For example, this rate was 1.5\% in Malawi (12), 23.1\% in Romania (13), and 43\% in Egypt (14).

As known, $10 \%$ to $15 \%$ of patients with HBV progress to cirrhosis within two years; the rate is $70 \%$ to $80 \%$ within 5 to 10 years (15). In our study, the prevalence of liver cirrhosis was $46.25 \%$, which is a significant rate of cirrhosis in these patients. Meanwhile, HDV in different individuals may show different disease courses from mild to severely progressive (16). Our study revealed a maximum of $62 \mathrm{KP}$ and a minimum of $6 \mathrm{KP}$ in patients' Fibroscan, with different stages of progression. This result was similar to the liver biopsy in our patients. Our study revealed that the prevalence of HCC in patients with HBV and HDV infection was $8.75 \%$. Makhlouf et al. (14), in their study in Egypt, reported $8.8 \%$ of patients with HCC, and another study showed that the rate of HCC in hepatitis B and D co-infection is almost three times the rate among HBV mono-infection (17). In the Miao et al. (11) meta-analysis, the odds ratios for co-infected patients for being asymptomatic, having a diagnosis of cirrhosis, or having HCC or mortality were 0.12 (95\% CI: 0.06 - 0.21), 3.90 (95\% CI: 2.94 -
5.18), and 1.97 (95\% CI:1.02 - 3.78), respectively.

The HDV RNA PCR was positive in $38.75 \%$ of the patients in our research while it was $16.4 \%$ in a study in Romania (13) and 31.3\% in Egypt (14). We could not find a significant correlation between hepatic cirrhosis and HBV DNA viral load; however, there was an association between liver cirrhosis and HDV RNA viral load. Therefore, patients with positive HDV RNA are more susceptible to cirrhosis than patients with undetectable HDV RNA. It was demonstrated that persistent HDV replication is associated with cirrhosis development (18).

In our research, $37.5 \%(n=30)$ of the patients were treated with interferon; 14 (14/30, 46.6\%) patients completed interferon therapy (for at least 48 weeks) and showed response with undetectable HDV RNA PCR after treatment, but none of them had a cirrhotic liver. The virological response is seen in no more than $50 \%$ of the patients (16); in our center, the response was about 50\%. However, it should be mentioned that seven $(7 / 30,23.33 \%)$ patients did not come to the clinic to continue treatment. Also, our study revealed that treatment with interferon and response to it can save the liver from failure.

The limitation of our study was the lack of exact followup information of patients after treatment and that all patients were chronic HBV and HDV patients, and we could not determine co-infection or super-infection of HDV. Besides, the way of disease transmission was indeterminate.

\section{Conclusions}

The HBV and HDV co-infected is the most important infection among types of viral hepatitis, and if diagnosed with delay, it can result in liver failure and malignancy. According to our results regarding cirrhotic patients with HBV/HDV co-infection with a rate of about $46 \%$, it seems that the early detection of HDV Ab has a significant role in the prevention of progressive liver failure, and treatment with interferon can help these patients to have a good prognosis.

\section{Footnotes}

Authors' Contribution: $\mathrm{MH}$ drafted the manuscript. ZA and JB performed data gathering. MQ performed statistical analyses. FG supervised the study and edited the manuscript for intellectual content.

Conflict of Interests: All authors declare that they have no relevant financial interests and conflict of interest.

Funding/Support: This research project was fully sponsored by the Tehran University of Medical Sciences with grant number 33743. 
Informed Consent: Written informed consent was obtained from all participants before the initiation of the study.

\section{References}

1. Rizzetto M. Hepatitis D Virus: Introduction and Epidemiology. Cold Spring Harb Perspect Med. 2015;5(7). a021576. doi: 10.1101/cshperspect.a021576. [PubMed: 26134842]. [PubMed Central: PMC4484953].

2. Schweitzer A, Horn J, Mikolajczyk RT, Krause G, Ott JJ. Estimations of worldwide prevalence of chronic hepatitis B virus infection: a systematic review of data published between 1965 and 2013. Lancet. 2015;386(10003):1546-55. doi: 10.1016/s0140-6736(15)61412-x.

3. Ataei B, Yazdani MR, Kalantari H, Yaran M, Nokhodian Z, Javadi AA, et al. Hepatitis D virus infection in Isfahan, central Iran: Prevalence and risk factors among chronic HBV infection cases. Hepat Mon. 2011;11(4):269-72. [PubMed: 22706272]. [PubMed Central: PMC3206699].

4. Chen HY, Shen DT, Ji DZ, Han PC, Zhang WM, Ma JF, et al. Prevalence and burden of hepatitis D virus infection in the global population: a systematic review and meta-analysis. Gut. 2019;68(3):512-21. doi: 10.1136/gutjnl-2018-316601. [PubMed: 30228220].

5. Plesniak R, Wawrzynowicz-Syczewska M. Prevalence of hepatitis delta infections among HBs-antigen-positive inhabitants of southeastern and northwestern parts of Poland. Clin Exp Hepatol. 2019;5(3):232-6. doi: 10.5114/ceh.2019.87636. [PubMed: 31598560]. [PubMed Central: PMC6781819].

6. Bruce MG, Bruden D, Hurlburt D, Zanis C, Thompson G, Rea L, et al. Antibody Levels and Protection After Hepatitis B Vaccine: Results of a 30-Year Follow-up Study and Response to a Booster Dose. J Infect Dis. 2016;214(1):16-22. doi: 10.1093/infdis/jiv748. [PubMed: 26802139].

7. Abbas Z, Abbas M, Abbas S, Shazi L. Hepatitis D and hepatocellular carcinoma. World JHepatol.2015;7(5):777-86. doi: 10.4254/wjh.v7.i5.777. [PubMed: 25914778]. [PubMed Central: PMC4404383].

8. Hughes SA, Wedemeyer H, Harrison PM. Hepatitis delta virus. Lancet. 2011;378(9785):73-85. doi: 10.1016/S0140-6736(10)61931-9. [PubMed: 21511329].
9. Lempp FA, Ni Y, Urban S. Hepatitis delta virus: insights into a peculiar pathogen and novel treatment options. Nat Rev Gastroentero Hepatol. 2016;13(10):580-9. doi: 10.1038/nrgastro.2016.126. [PubMed 27534692].

10. Elsaid MI, Li Y, John T, Narayanan N, Catalano C, Rustgi VK. Economic and Health Care Burdens of Hepatitis Delta: A Study of Commercially Insured Adults in the United States. Hepatology. 2020;72(2):399-411. doi: 10.1002/hep.31055. [PubMed: 31804707].

11. Miao Z, Zhang S, Ou X, Li S, Ma Z, Wang W, et al. Estimating the Global Prevalence, Disease Progression, and Clinical Outcome of Hepatitis Delta Virus Infection. J Infect Dis. 2020;221(10):167787. doi: 10.1093/infdis/jiz633. [PubMed: 31778167]. [PubMed Central: PMC7184909].

12. Stockdale AJ, Mitambo C, Everett D, Geretti AM, Gordon MA. Epidemiology of hepatitis B, C and D in Malawi: systematic review. BMC Infect Dis. 2018;18(1):516. doi: 10.1186/s12879-018-3428-7. [PubMed: 30314448] [PubMed Central: PMC6186098].

13. Gheorghe L, Csiki IE, Iacob S, Gheorghe C, Trifan A, Grigorescu $M$, et al. Hepatitis Delta Virus Infection in Romania: Prevalence and Risk Factors. J Gastrointestin Liver Dis. 2015;24(4):413-21. doi 10.15403/jgld.2014.1121.244.dtv. [PubMed: 26697566].

14. Makhlouf NA, Morsy KH, Mahmoud AA. Hepatitis D virus infection among hepatitis B virus surface antigen positive individuals in Upper Egypt: Prevalence and clinical features. J Infect Public Health. 2019;12(3):350-6. doi: 10.1016/j.jiph.2018.12.001. [PubMed: 30833193].

15. Koh C, Da BL, Glenn JS. HBV/HDV Coinfection: A Challenge for Therapeutics. Clin Liver Dis. 2019;23(3):557-72. doi: 10.1016/j.cld.2019.04.005. [PubMed: 31266627]. [PubMed Central: PMC6659751].

16. Yurdaydin C, Idilman R, Bozkaya H, Bozdayi AM. Natural history and treatment of chronic delta hepatitis. J Viral Hepat. 2010;17(11):749-56. doi: 10.1111/j.1365-2893.2010.01353.x. [PubMed: 20723036].

17. Brancaccio G, Fasano M, Grossi A, Santantonio TA, Gaeta GB. Clinical outcomes in patients with hepatitis $\mathrm{D}$, cirrhosis and persistent hepatitis B virus replication, and receiving long-term tenofovir or entecavir. Aliment Pharmacol Ther. 2019;49(8):1071-6. doi: 10.1111/apt.15188. [PubMed: 30793345].

18. Romeo R, Petruzziello A, Pecheur EI, Facchetti F, Perbellini R, Galmozzi E, et al. Hepatitis delta virus and hepatocellular carcinoma: an update. Epidemiol Infect. 2018;146(13):1612-8. doi: 10.1017/S0950268818001942. [PubMed: 29991359]. 